\title{
BILDUNG UND ERZIEHUNG
}

Begründet von Franz Hilker(†)

Herausgeber*innen:

Prof. Dr. Karin Büchter, Prof. Dr. Manfred Heinemann, Prof. (H) Dr. Heribert Hinzen, Prof. Dr. Hilmar Hoffmann, Prof. Dr. Hans-Georg Kotthoff, Prof. Dr. Eva Matthes (Sprecherin),

Prof. Dr. Elisabeth Meilhammer, Prof. Dr. Gisela Miller-Kipp, Prof. Dr. Harm Paschen, Prof. Dr. Anatoli Rakhkochkine, Prof. Dr. Una Röhr-Sendlmeier, Prof. Dr. Sonja Steier.

74. JAHRGANG 2021

Heft 1: Aktuelle Forschungen zu Erziehung und Bildung, hg. von Hans-Georg Kotthoff / Anatoli Rakhkochkine Heft 2: Professionalisierung frühkindlicher Bildung am Scheideweg?,

hg. von Hilmar Hoffmann / Eva Matthes

Heft 3: Künstliche Intelligenz und Pädagogik, hg. von Gisela Miller-Kipp / Anatoli Rakhkochkine

Heft 4: Inklusion. Waldorfpädagogik und Erziehungswissenschaft im Gespräch, hg. von Eva Matthes / Jakob Benecke 
ISSN (Printausgabe): 0006-2456, ISSN (online): 2194-3834

(C) 2021 by Vandenhoeck \& Ruprecht, an imprint of the Brill-Group (Koninklijke Brill NV, Leiden, The Netherlands; Brill USA Inc., Boston MA, USA; Brill Asia Pte Ltd, Singapore; Brill Deutschland GmbH, Paderborn, Germany; Brill Österreich GmbH, Vienna, Austria).

Koninklijke Brill NV incorporates the imprints Brill, Brill Nijhoff, Brill Hotei, Brill Schöningh, Brill Fink, Brill mentis, Vandenhoeck \& Ruprecht, Böhlau, Verlag Antike, V\&R unipress.

Verlag: Brill Deutschland GmbH, Theaterstr. 13, D-37073 Göttingen Alle Rechte vorbehalten. Das Werk und seine Teile sind urheberrechtlich geschützt. Jede Verwertung in anderen als den gesetzlich zugelassenen Fällen bedarf der vorherigen schriftlichen Einwilligung des Verlages. Satz: $3 w+p$, Rimpar 


\section{Register}

\section{SCHWERPUNKTTHEMEN}

Hilmar Hoffmann, Professionalisierung frühkindlicher Bildung am Scheideweg - Einleitung zu diesem Heft

Hans-Georg Kotthoff / Anatoli Rakhkochkine, Einleitung .......................................................

Eva Matthes / Jakob Benecke, Einleitung ...................................................................... Künstliche Intelligenz und Pädagogik. Zur Einleitung in dieses Heft

\section{BEITRÄGE}

Cristina Alarcón, Das typische Gymnasialkind. Optimierung der Passung zwischen Grundschule und Gymnasium durch Grundschulgutachten in der jungen Bundesrepublik

Ulrike Barth / Thomas Maschke, Inklusion und Waldorfpädagogik: Konkretisierungen und kritische Überlegungen

Johannes Bellmann / Katharina Hans / Judith Küper / Corrie Thiel, Qualität als Tradierungsproblem. Eine Auseinandersetzung mit Forschungsperspektiven auf evaluative Praktiken in der Lehrer*innenbildung

Saskia Bender, Soziale Ordnung in alternativen Schulen. Zum inklusiven Anspruch der Waldorfpädagogik

Peter Cloos / Edita Jung, Kindheitspädagogische Qualifizierung an Hochschulen Zwischen den Erwartungshorizonten und Realitäten des frühpädagogischen Feldes

Georg Feuser, Inklusion und Waldorfpädagogik. Eine allgemeindidaktische Perspektive

Ulrich Heimlich, Waldorfpädagogik als Inklusive Pädagogik? Chancen und Barrieren eines Dialogs

Anke Karber, Praktiken der Reflexion in pädagogischen Qualifizierungen - Forschungsund Entwicklungsanfragen

Eleftherios Klerides, The notions of power and change in the historiography of education in Cyprus: Simplifications and possibilities for new directions

Melanie Kubandt, Geschlechterrelevanzen im Hinblick auf pädagogische Fachkräfte in Kindertageseinrichtungen: Anforderungen für die Ausbildung

Stefan Küchemann / Andreas Dengel / Jochen Kuhn, Künstliche Intelligenz im LehrLernprozess von MINT-Fächern: Vom adaptiven Lernmaterial zur Implementation in die Lehrkräftebildung

Rebecca Lazarides / Johann Chevalére, Artificial intelligence and education: Addressing the variability in learners' emotion and motivation with adaptive teaching assistants

Junmin Li / Matthias Pilz / Christian Gronowski, Lernaufgaben in der Hochschuldidaktik: Eine Untersuchung zum Einsatz von Lernaufgaben

Manolis Mavrikis / Mutlu Cukurova / Daniele Di Mitri / Jan Schneider / Hendrik Drachsler, A short history, emerging challenges and co-operation structures for Artificial Intelligence in education

Anna Schlomann / Christian Rietz / Peter Zentel / Vera Heyl / Hans-Werner Wahl, KIbasierte Sprachassistenz im Licht der Heterogenität von Altern: Das Beispiel geistige Behinderung

Albert Schmelzer, Grundlagen inklusiver Waldorf-Schulpädagogik 
Elisa Tessmer / Maximilian Schäfer, Lehramtsstudium der Beruflichen Fachrichtung Sozialpädagogik - Status quo und aktuelle Entwicklungen in Zeiten des Lehrkräftemangels

Sven Thiersch / Eike Wolf, Schule zwischen Digitalisierung und Disziplinierung. Rekonstruktionen pädagogischer Generationsbeziehungen im digitalisierten Unterricht

Maria Wirzberger / Madeleine Schwarz, Förderung selbstregulierten Lernens durch ein KI-gestütztes Training

Christoph Wulf, Digitale Transformation und Künstliche Intelligenz im Anthropozän

Christian Timo Zenke / Benedict Kurz, School as an „experimental station“: Über das Prinzip der Laboratory School und seine Verbreitung in Europa

\section{ForUM}

Bernd Ahrbeck, „Behinderung gibt es nicht.“ Oder vielleicht doch? Überlegungen zum schulischen Inklusionsdiskurs

Kirsten Fuchs-Rechlin / Thomas Rauschenbach, Erzieher*innen - ein Qualifikationsprofil in der Zwickmühle. Seitenwege, Irrwege, Auswege

Frank Tosch, Das besondere Exponat: LC 80 - der erste Lerncomputer der DDR

\section{Rezensionen}

Ulrich Herrmann (Hrsg.) (2019): Pädagogische Beziehungen. Grundlagen - Praxisformen - Wirkungen (Gisela Miller-Kipp)

Stefan Klusemann / Lena Rosenkranz / Julia Schütz (2020): Professionelles Handeln im System. Perspektiven pädagogischer Akteur*innen auf die Personalsituation in Kindertageseinrichtungen (HiSKiTa) (Silke Antoni)

Knox, Jeremy / Wang, Yuchen / Gallagher, Michael (eds.) (2019): Artificial Intelligence and Inclusive Education: Speculative Futures and Emerging Practices (Anatoli Rakhkochkine)

Thomas Maschke (Hrsg.) (2019): Bildungsinnovation: Impulse aus Reformpädagogik und Inklusiver Pädagogik (Alexandra Schotte)

Ingrid Miethe / Tim Kaiser / Tobias Kriele / Alexandra Piepiorka (2019): Globalisation of an Educational Idea: Workers' Faculties in Eastern Germany, Vietnam, Cuba and Mozambique (Anatoli Rakhkochkine)

Barbara Schellhammer / Berthold Goerdele (Hrsg.) (2020): Bildung zum Widerstand (Franz Gmainer-Pranzl)

Heinz-Elmar Tenorth (2020): Die Rede von Bildung. Tradition, Praxis, Geltung - Beobachtungen aus der Distanz (Gisela Miller-Kipp) 